\begin{tabular}{|c|l|}
\hline Title & $\begin{array}{l}\text { Identification of the Precise Kairomone sensitive Period and Histological Characterization of Necktooth Formation in } \\
\text { Predator-induced Poly phenism in Daphnia pulex }\end{array}$ \\
\hline Author(s) & Naraki, Y uka; Hiruta, Chizue; Tochinai, Shin \\
\hline Citation & $\begin{array}{l}\text { Zoological Science, 30(8), 619-625 } \\
\text { https://doi.org/40.2108/2s.30.619 }\end{array}$ \\
\hline Issue Date & 2013.08 \\
\hline Doc URL & http:/hdl.handle.net/2115/56643 \\
\hline Type & article \\
\hline File Information & zsi.30.619.pdf \\
\hline
\end{tabular}

Instructions for use 


\title{
Identification of the Precise Kairomone-sensitive Period and Histological Characterization of Necktooth Formation in Predator-induced Polyphenism in Daphnia pulex
}

\author{
Yuka Naraki $^{1 \dagger}$, Chizue Hiruta ${ }^{2 \star \dagger}$, and Shin Tochinai ${ }^{1}$ \\ ${ }^{1}$ Department of Natural History Sciences, Faculty of Science, Hokkaido University, \\ Kita 10 Nishi 8, Kita-ku, Sapporo, Hokkaido 060-0810, Japan \\ ${ }^{2}$ Okazaki Institute for Integrative Bioscience, National Institute of National Sciences, \\ Higashiyama 5-1, Myodaiji, Okazaki, Aichi 444-8787, Japan
}

\begin{abstract}
Many organisms have the ability to alter their development in the presence of predators, leading to predator-induced defenses that reduce vulnerability to predation. In the water flea Daphnia pulex, small protuberances called 'neckteeth' form in the dorsal neck region in response to kairomone(s) released by predatory phantom midges (Chaoborus larvae). Although previous studies suggested that kairomone sensitivity begins when chemoreceptors begin to function during embryogenesis, the exact critical period was unknown to date. In this study, we investigated the period of kairomone sensitivity and the process of necktooth formation in $D$. pulex through extensive treatments with pulses of kairomone(s). First, we described the time course of embryogenesis, which we suggest should be used as the standard in future studies. We found the kairomone-sensitive period to be relatively short, extending from embryonic stage 4 to postembryonic first instar. We observed cell proliferation and changes in cell structure in response to the kairomone treatment, and propose a model for necktooth formation. Preliminary $\mathrm{LiCl}$ treatment suggests the Wnt signaling pathway involved in crest formation as a candidate for the molecular mechanism underlying this process. Our study provides basic insight toward understanding the mechanisms underlying adaptive polyphenism in $D$. pulex.
\end{abstract}

Key words: Daphnia pulex, neckteeth, predator-induced defense, kairomone(s), phenotypic plasticity, polyphenism

\section{INTRODUCTION}

Many organisms are able to change their morphology and/or behavior in response to the threat of predation. The phenotypic alteration by modulating developmental processes in the presence of predators is called "predatorinduced defense" or "predator-induced polyphenism," and these modifications can increase the fitness of prey organisms (Gilbert, 2010). Various members of the cladoceran genus Daphnia (commonly called "water fleas") display a variety of phenotypic changes, mainly in head shape (Tollrian and Harvell, 1999). Of these, the best-studied system is the induction of neckteeth in $D$. pulex by a kairomone (or kairomones) released by predatory phantom midges, Chaoborus larvae (Tollrian and Harvell, 1999). A kairomone is a class of 'semiochemical,' which refers to chemicals or mixtures of chemicals that act as messengers within or between species. Kairomones are substances, released into the environment by an organism, which mediate reactions in another organism in such a way that the recipient rather than the

\footnotetext{
* Corresponding author. Tel. : :+81-564-59-5238;

Fax : +81-564-59-5236;

E-mail: chizueh@gmail.com

† These authors equally contributed to this study. doi:10.2108/zsj.30.619
}

emitter receives benefit (Grasswitz and Jones, 2002).

Daphnia species are important in pond and lake ecosystems, and their field ecology has been studied extensively (Dodson et al., 2009). Daphnia pulex is a potentially useful model organism for studying predator-induced polyphenism at the genetic and molecular levels (Stollewerk, 2010), for several reasons. It was the first crustacean to have its genome sequenced (Colbourne et al., 2011), and a genetic linkage map has been constructed (Cristescu et al., 2006). In addition, molecular techniques, such as in situ hybridization and RNAi, have now been extended to daphnid species (Sagawa et al., 2005; Kato et al., 2011). Finally, individuals with the same genetic background can express a variety of phenotypes in response to environmental cues (Tollrian and Harvell, 1999). Interdisciplinary studies of predator-induced polyphenism in $D$. pulex thus have the potential to elucidate mechanisms underlying the evolution of plasticity (Jenner and Wills, 2007; Tollrian and Leese, 2010).

Morphogenesis of neckteeth appears to begin during embryogenesis (Laforsch and Tollrian, 2004; Imai et al., 2009). Previous studies have suggested that, in $D$. pulex, kairomone reception during embryonic development is required for necktooth induction (Krueger and Dodson, 1981; Parejko, 1992), and Imai et al. (2009) reported that exposure to a kairomone after the first-instar juvenile stage is necessary to maintain the neckteeth. However, it is 
unknown whether the ability to respond to kairomone(s) is limited to a certain period of embryonic development. Likewise, although Beaton and Hebert (1997) reported that polyploid cells at the region of necktooth formation may be involved in the formation of neckteeth, little is known about the histological basis underlying the development of the defensive morph. Recently, candidate molecular pathways have been identified that appear to be primarily involved in morphological defense formation in $D$. pulex (Miyakawa et al., 2010; Spanier et al., 2010), but additional histological and morphological information are still needed to fully interpret these molecular studies.

In the present study, we applied pulse treatments of predator-conditioned medium of varying duration and intervals to $D$. pulex during the embryonic and early juvenile stages to detect the period of sensitivity to kairomones. We observed the uptake of dextran-tetramethylrhodamine during embryogenesis to determine when embryos absorb external solutions, possibly including kairomone(s). Using BrdU (5'-bromo-2'-deoxyuridine), we also observed the timing of cell proliferation in the occipital region during necktooth formation. Finally, to obtain clues regarding the molecular mechanisms underlying defense morph formation, we focused on Wnt pathways, which play a key role in several steps in animal development, such as in establishing the polarity of insect and vertebrate limbs and in stem-cell proliferation (Gilbert, 2010; Goto and Hayashi, 1999). We treated animals with $\mathrm{LiCl}$, which inhibits glycogen synthase kinase $3 \beta$ and affects the transcription of Wnt target genes (Klein and Melton, 1996).

\section{MATERIALS AND METHODS}

\section{Animals}

A single clone of $D$. pulex, which had been collected from a pond at the Hokkaido University campus, Sapporo, Japan, was used throughout this study. Animals were maintained in dechlorinated tap water at $18^{\circ} \mathrm{C}$ under artificial light conditions of $14 \mathrm{~h}$ light and $10 \mathrm{~h}$ dark to induce and maintain reproduction. They were fed with a concentrated monoculture of the green alga Chlamydomonas reinhardtii, as described in Sueoka (1960). In all experiments, $D$. pulex embryos were collected from dissected maternal brood chambers and cultured, as previously reported (Imai et al., 2009). We defined embryonic stages using the scheme of Laforsch and Tollrian (2004).

Fourth-instar Chaoborus flavicans larvae were collected from a pond at the National Institute for Environmental Studies, Tsukuba, Japan.

\section{Kairomone medium}

Chaoborus larvae were cultured in dechlorinated tap water at a density of 1-5 larvae/100 $\mathrm{ml}$ for one week in a temperature- and light-controlled incubator $\left(18^{\circ} \mathrm{C} ; 14 \mathrm{~h}\right.$ light and $10 \mathrm{~h}$ dark), and were fed daily with sufficient $D$. pulex. Water in which Chaoborus larvae had been incubated was passed through a $1.2 \mu \mathrm{m}$ filter (RAWPO4700; Millipore), dispensed into a $15 \mathrm{ml}$ or $50 \mathrm{ml}$ conical tube (352097 and 352098; BD Falcon), and then stored at $-20^{\circ} \mathrm{C}$. After thawing at room temperature, the water was filtered through a Whatman GF/C filter before use; this Chaoborus-conditioned water is hereafter referred to as 'kairomone medium' or 'kairomone'. Dechlorinated tap water was used as the control medium. We evaluated kairomone quality before and after freezing, and only used kairomone in which the necktooth induction rate was at least $80 \%$ when embryos were reared in undiluted kairomone medium.

\section{Induction of defenses}

Juveniles reared in kairomone medium were examined at each instar under a stereomicroscope, and we recorded whether they had formed neckteeth, and how the neckteeth formed. The chisquare test was used to assess differences in the proportions of individuals with neckteeth between kairomone-treated and control animals.

For kairomone pulse treatments, 48-well culture plates (353078; BD Falcon) filled with $1 \mathrm{ml}$ of kairomone or control medium per well were used. Embryos or juveniles were transferred individually (one animal/well) from a well containing control medium to one containing kairomone medium. Whenever embryos or juveniles were transferred from kairomone into control medium, they were first rinsed five times in control medium in a $60 \mathrm{~mm}$ plastic dish (353002; BD Falcon). At first, we designed induction experiments in which embryos and juveniles were exposed to kairomone to reveal which stage is important to induce neckteeth (Fig. $3 \mathrm{~A}-\mathrm{C}$ and $\mathrm{F}-\mathrm{V}$ ). After obtaining the results, we then conducted the remaining treatments to confirm and highlight the importance of stage 4 (Fig. 3D, $E$ and $P A-P Q$ ). Second-instar animals were examined under a stereomicroscope, and we recorded whether they had formed neckteeth. The induction values cannot be compared between treatments because the kairomone concentration was not exactly the same within experiments.

\section{Hematoxylin and eosin (HE) staining}

Animals were fixed in Bouin's fluid, dehydrated in an ethanol series $(70 \%, 80 \%, 90 \%, 95 \%, 99 \%, 99 \%)$ and xylene twice for $30 \mathrm{~min}$ each, embedded in paraffin, and serially sectioned. The 5$\mu \mathrm{m}$ sections were deparaffinized with xylene three times for $5 \mathrm{~min}$ each, transferred to an ethanol series $(99 \%, 99 \%, 95 \%, 90 \%, 80 \%$, $70 \%$ ) for 5 min each, and then stained with Delafield's hematoxylin and eosin.

\section{Scanning electron microscopy (SEM)}

For SEM, a method modified from Laforsch and Tollrian (2000) was used. Animals were killed by exposure to $600 \mathrm{~W}$ microwave radiation for $7 \mathrm{sec}$ and immediately transferred into $70 \%$ ethanol for $3 \mathrm{~h}$. Fixed specimens were dehydrated in an acetone series $(70 \%$, $80 \%, 90 \%, 95 \%, 99 \%, 99 \%$ ) for 10 min each, incubated in hexamethyldisilazane for $70 \mathrm{~min}$, and transferred to a desiccator. Dried specimens were glued to a brass holder and gold-coated for observation with a S3000N scanning electron microscope (HITACHI).

\section{Uptake of dextran-tetramethylrhodamine}

Embryos were soaked in $0.1 \mathrm{mg} / \mathrm{ml}$ dextran-tetramethylrhodamine (Invitrogen) for $1 \mathrm{~h}$ during embryonic stage 3 (Fig. $4 \mathrm{~A}$ ), for $2 \mathrm{~h}$ during stages 3 and 4 (Fig. $4 \mathrm{~B}$ ), or for 30 min during embryonic stage 4 (Fig. 4C). They were then washed in dechlorinated tap water three times and observed as whole mount preparations under a fluorescence microscope (BX-50, BX-FLA; Olympus).

\section{BrdU immunohistochemistry}

Starting in each developmental stage, animals were incubated in kairomone or control medium containing $5 \mathrm{mM}$ BrdU (5'-bromo2'-deoxyuridine; SIGMA) for $20 \mathrm{~h}$ prior to fixation (Fig. 5A). After treatment, they were fixed in Carnoy's solution (1:3 acetic acid to methanol) at room temperature for $15 \mathrm{~min}$, dehydrated in ethanol and xylene, embedded in paraffin, and serially sectioned. The 5- $\mu \mathrm{m}$ sections were then rehydrated, washed in $0.1 \%$ Triton X-100 in Phosphate-buffered saline (PBS), treated with $2 \mathrm{~N} \mathrm{HCl}$ for $20 \mathrm{~min}$, washed again in PBS, and incubated in a blocking solution of $10 \%$ fetal calf serum (FCS) for $15 \mathrm{~min}$, followed by rat monoclonal antiBrdU antibody (1:250 in blocking solution; Abcam) for $2 \mathrm{~h}$. After another wash in PBS, the sections were incubated with Cy3conjugated anti-rat IgG goat antibody (1:500; Jackson ImmunoResearch) for $1 \mathrm{~h}$ at room temperature. After three washes in PBS 
(5 min/wash), the sections were stained with $1 \mu \mathrm{g} / \mathrm{ml}$ Hoechst 33342 (Molecular Probes) in PBS for $5 \mathrm{~min}$ to visualize nuclear DNA, and then washed three times ( $5 \mathrm{~min} /$ wash) in PBS. Finally, the sections were mounted in VECTASHIELD Mounting Medium $\mathrm{H}-1000$ (Vector) and observed under a fluorescence microscope (BX-50, BX-FLA; Olympus). The number of BrdU-positive cells per necktooth-forming region was compared between kairomonetreated and control animals, using the $t$-test.

\section{LiCl treatment}

Animals were incubated from stage 1 embryos to the juvenile stage in kairomone or control medium containing $3 \mathrm{mM} \mathrm{LiCl}$ (Nacalai Tesque), a well-known inhibitor of glycogen synthase kinase $3 \beta$ in the Wnt signal transduction cascade (Klein and Melton, 1996), and observed daily under a stereomicroscope.

\section{RESULTS}

\section{Time course of embryogenesis and early post- embryogenesis}

Figure 1 shows the time course of embryogenesis and early post-embryogenesis, which proceeded along a strict time course at $18^{\circ} \mathrm{C}$. Although embryos within a clutch developed at nearly the same pace, the timing of hatching from the egg chorion varied somewhat, and so we designated the time at which half the embryos in a clutch had hatched as $0 \mathrm{~h}$ on the time scale. In embryonic stage 1, which is the interval from oviposition to hatching $(0 \mathrm{~h})$, the egg was covered by three membranes: the egg chorion (the outer-most membrane), and the second and third membranes underlying it. In stage 2, lasting from $0 \mathrm{~h}$ to $24 \mathrm{~h}$, the embryo remained covered by the second and third membranes. In stage 3, lasting from $24 \mathrm{~h}$ to $30 \mathrm{~h}$, both of these membranes began to peel off, and immature organs such as first antennae became visible. In stage 4 , lasting from $30 \mathrm{~h}$ to $42 \mathrm{~h}$, the appendages appeared mature and became functional, and the animals could swim with their second antennae. Around $42 \mathrm{~h}$, the animals completely shed the third membrane, changed in shape from the embryonic to the adult form, and developed into first-instar juveniles. One of the characteristic features in the first-instar was complete elongation of the tail spine. By three days after hatching (after 72 h) many individuals had reached the second-instar stage.

\section{Defense morph induction}

Figure 2A shows a second-instar juvenile lacking neckteeth. The neckteeth (Fig. 2B, C) comprise several tiny spikes alternately arranged (Fig. 2D) in two rows on each side of the dorsal carapace ridge between the insertion points of the first and second antennal muscles. The epithelium at the base of the neckteeth becomes thickened to form a structure called the "crest." Some control juveniles in the first instar, and very few in the second instar, developed neckteeth, although they were reared in control medium (Fig. 2E), which is in agreement with Tollrian (1993). Juve-
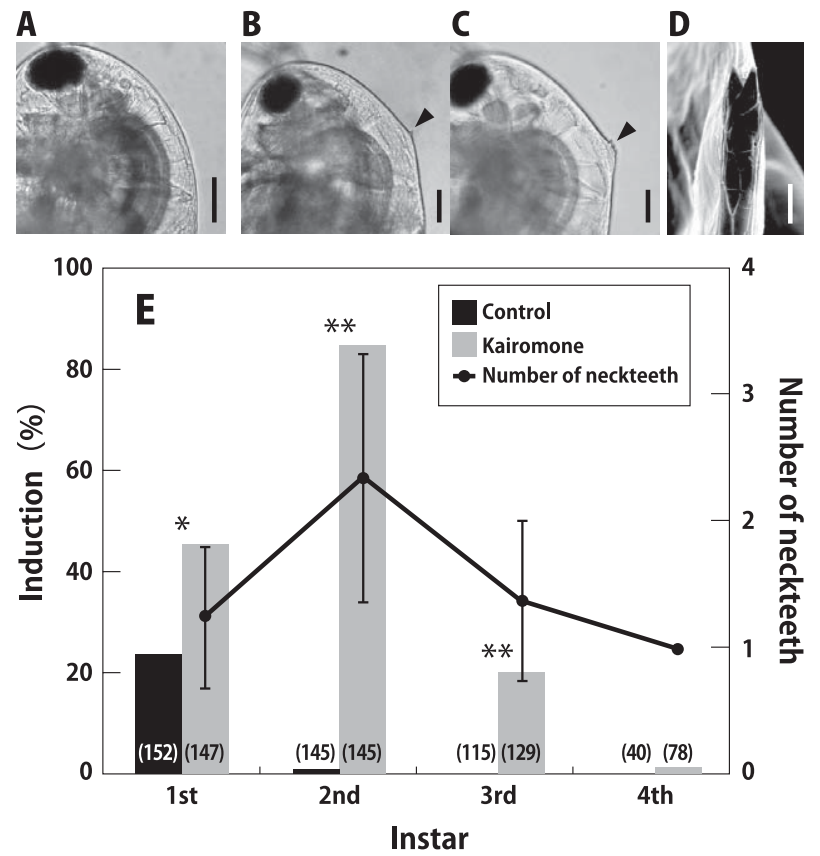

Fig. 2. (A) A second-instar juvenile lacking neckteeth. (B) Firstand (C) second-instar juvenile with neckteeth (arrowheads). (D) SEM image showing neckteeth in dorsal view. Scale bars: $50 \mu \mathrm{m}$ (A-C), $10 \mu \mathrm{m}$ (D). (E) Induction frequency (bars) and number of neckteeth (line) at each instar. The proportion of individuals bearing neckteeth was compared between treated animals exposed to kairomone medium (kairomone treatment) and controls exposed to dechlorinated tap water (asterisks indicate a significant difference between treated and control animals; ${ }^{*} ; P<0.05$, ${ }^{\star \star} ; P<0.005$, chisquare test). Data are shown as mean values \pm SD. Numbers in parentheses indicate the sample size.

niles reared in kairomone medium produced neckteeth from the first to the fourth instar stages (Fig. 2B-D), with the second-instar stage having the highest values for both the frequency of occurrence of neckteeth and the average number of spikes (Fig. 2E). From these results, we assessed kairomone sensitivity for defense morph induction by the scores counted in the second instar juveniles.

To determine the kairomone-sensitive period, embryos and juveniles were incubated in kairomone medium at different intervals and for different durations (Fig. 3). Long exposures from stage 2 to the first instar tended to increase the induction of neckteeth (Fig. 3, rows A-C). In contrast, ani- 


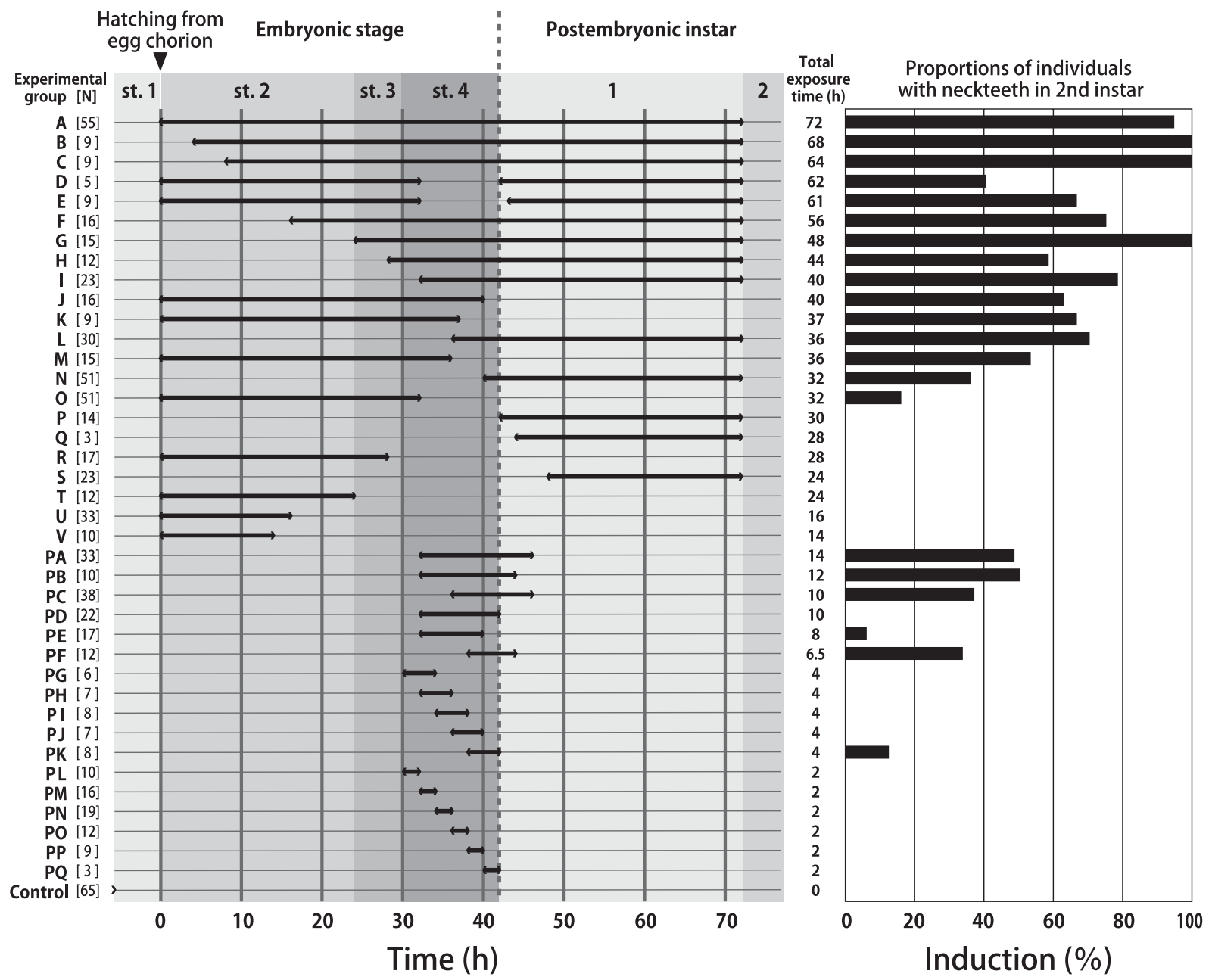

Fig. 3. Kairomone pulse treatments of various durations from embryonic stage 2 through the first instar. Heavy horizontal lines represent periods of kairomone treatment. Induction frequency of neckteeth scored in the second instar.

mals exposed to kairomone(s) only earlier or later than stage 4 developed no neckteeth (Fig. 3, rows P-V). Despite a long treatment interval, animals transferred from kairomone medium to the control medium during embryonic stage 4 showed a reduced frequency of necktooth formation (Fig. 3, rows D, E). Animals treated with kairomone(s) during and around embryonic stage 4 for a short period (Fig. 3, rows $P A-P Q$ ) tended to form neckteeth when they had been exposed to kairomone(s) toward the end of this stage (Fig. 3, rows PA, PB, PC, PF, at around 40-42 h). Moreover, even when embryos were treated for the same $4 \mathrm{~h}$ during embryonic stage 4, the early treatment induced no neckteeth (Fig. 3, rows PG-PJ) in contrast to the late treatment (Fig. 3, row PK).

To examine whether embryos acquired the ability to absorb external solutions, presumably including kairomones, at stage 4, we examined the uptake of fluorescent dextran during embryonic stages 3 and 4 . We did not observe dye uptake in stage 3 embryos, but did in a stage 4 embryo. At stage 4, fluorescence was observed in the first antennae, dorsal organ, and intestine (Fig. 4). The results suggest that embryos hardly absorb kairomone at all in stage 3 , but readily absorbed it in stage 4 .
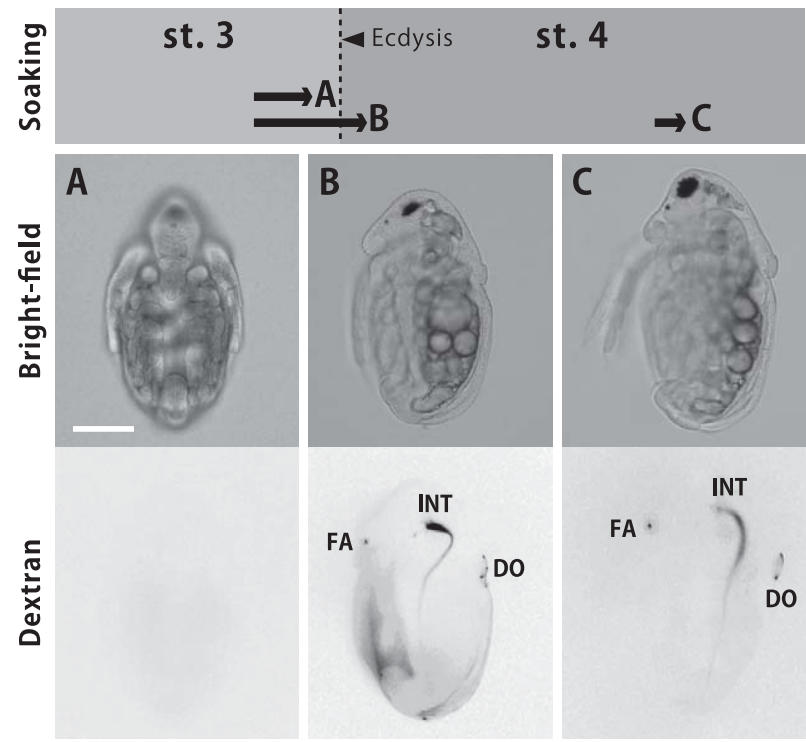

Fig. 4. Water influx into embryos detected by dextran-tetramethylrhodamine. Arrows indicate the treatment periods (A-C). FA, first antennae; INT, intestine; DO, dorsal organ. Scale bar $=20 \mu \mathrm{m}$. 
Immunohistochemical observation of cell proliferation in necktooth formation

To examine the contribution of cell proliferation to necktooth formation, we performed immunohistochemistry following BrdU incorporation (Fig. 5). No active cell proliferation was observed in parts of the body other than the region of necktooth formation. There was no significant difference in BrdUpositive cells/necktooth region between kairomone and control medium for Treatment I, confined to the embryonic stages. In contrast, epidermal cell proliferation was significantly higher in animals exposed to kairomone medium in postembryonic instars (Treatments II-V) than in the control. The number of BrdU-positive cells reached a peak in the second instar (Treatment V) following a decrease between the latter half of the firstinstar stage and the beginning of the second-instar stage (Treatment IV).

\section{Chemical treatments}

LiCl-free controls did not develop neckteeth (Fig. 6A, upper left). LiCl treatment without kairomone resulted in a slightly swollen crest-like structure without neckteeth (Fig. 6A, lower left). Although the number of neckteeth was not affected, the crest of neckteeth in kairomone and $3 \mathrm{mM} \mathrm{LiCl}$ treatment was more swollen in some individuals (Fig. 6A, lower right) compared to that in kairomone treatment without $\mathrm{LiCl}$ (Fig. 6A, upper right). Eight of 11 individuals subjected to the combined kairomone medium and $\mathrm{LiCl}$ treatment developed neckteeth, and five showed a highly swollen crest comprising of loose connective tissue (Fig. 6B right). All animals treated with $\mathrm{LiCl}$ died before reaching the second-instar stage.

\section{DISCUSSION}

\section{A developmental window for necktooth induction during late embryogenesis}

We found that the kairomone-sensitive period of $D$. pulex was embryonic stage 4 to first instar. This result is in agreement with previous studies that suggested the existence of a sensitive period during embryogenesis (Krueger and Dodson, 1981; Parejko, 1992; Imai et al., 2009). This critical period for necktooth induction in the $D$. pulex embryo can be viewed as a "developmental window." Animals treated with kairomone medium during this developmental window developed neckteeth, and the longer they were treated around the window, the more neckteeth they were likely to develop.

Under natural conditions, daphnids are subject to con- siderable environmental fluctuations (e.g., temperature, food concentration, predation risk) during embryogenesis and subsequent juvenile instars. In addition, necktooth formation has disadvantages, resulting in the avoidance of useless necktooth formation in the absence of predators (Havel and Dodson, 1987). Therefore, it is quite reasonable for daphnids that the developmental window is just prior to their release from the brood chamber, i.e., the time immediately prior to encountering predators.

\section{Kairomone action in D. pulex}

The fundamental assumption of our study is that a kairomone released by the predator into the ambient water constitutes the chemical cue for necktooth induction. Considering the pulse-treatment and dextran-tetramethylrhodamine experiments, we speculate that the kairomone is actively transported into the body through the first antennae, dorsal organ, and intestine mainly in embryonic stage 4, and may accumulate in the body during this stage. If kairomone dis- 

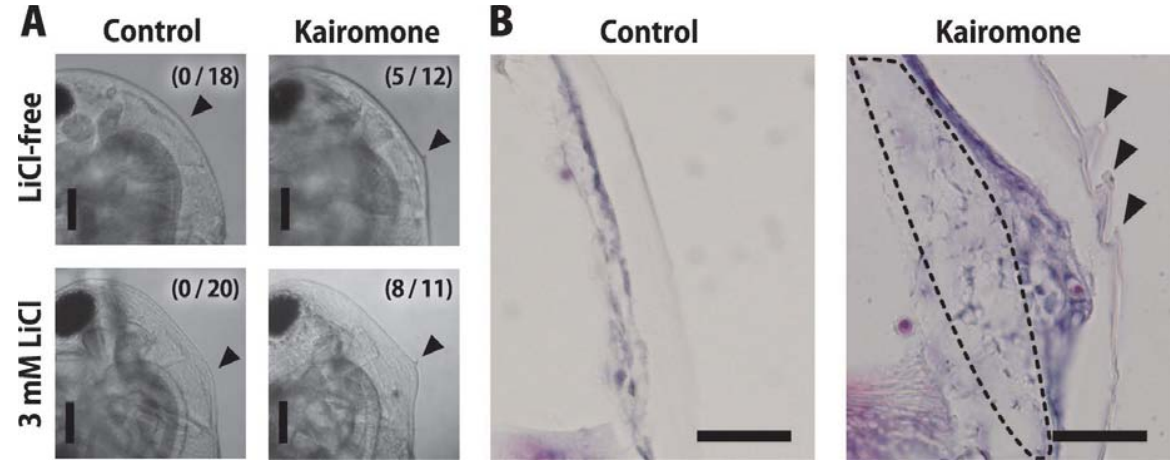

Fig. 6. (A) Combined treatment with kairomone and $\mathrm{LiCl}$. The fractions in parentheses indicate the proportion of individuals that formed neckteeth. Arrowheads indicate the site of necktooth formation. Scale bars $=50 \mu \mathrm{m}$. (B) Stained sagittal sections through the region where neckteeth form, from second-instar animals. Arrowheads indicate neckteeth; dashed outline indicates the crest. Scale bars $=20 \mu \mathrm{m}$.

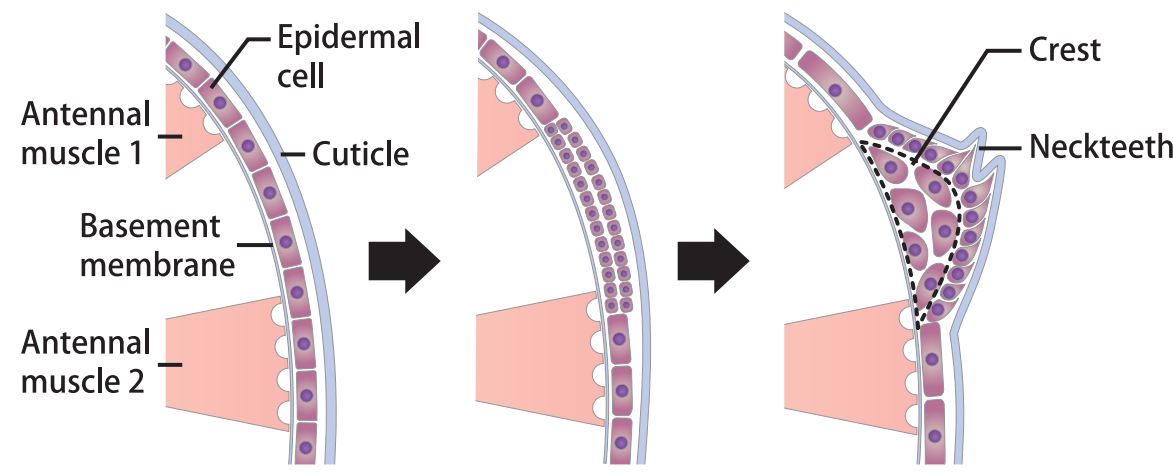

Fig. 7. Schematic diagram showing the process of necktooth formation (see Discussion).
Additionally, in some insects, it has been reported that plastic morphological traits are triggered by juvenile hormones (Miura, 2005; Suzuki and Nijhout, 2006). Thus, the endocrine system may be involved in initiating the development of neckteeth soon after the developmental window in $D$. pulex.

\section{Cellular changes during necktooth formation}

Necktooth spikes and the crest appear to be formed by different mechanisms, even though the same predator-released kairomone induces formation of both these defensive structures. In our study, BrdU immunohistochemistry showed that epidermal cells in the region of necktooth formation divided rapidly in postembryonic juveniles that had been exposed to kairomone, with the number of BrdUpositive cells in this region apparently correlated with necktooth formation. We observed that the crest consisted of loose connective tissue, whereas the epidermal cells lining the cuticle beneath the neckteeth were of high density and single-layered (Fig. 6B). Moreover, $\mathrm{LiCl}$ treatment indicated that $\mathrm{LiCl}$ affected only crest development rather than necktooth formation. appears from the environment, it appears that the kairomone stimulus is promptly lost from the $D$. pulex body. We hypothesize that the proportion of individuals that form neckteeth depends on the total amount of the stimulus received or accumulated at the end of embryogenesis. The results partly supporting these interpretations are as follows. In the experiment using fluorescently-labeled dextran, stage 3 embryos showed practically no uptake of the external solution despite $2 \mathrm{~h}$ of treatment. In contrast, the dye was detected in stage 4 embryos after just $30 \mathrm{~min}$ of treatment. After the third embryonic molt, the influx of various chemicals in the water appears to have increased, perhaps due because of the loss of physical barriers and/or alterations in permeability. Furthermore, since the Chaoborus kairomone (< 500 Daltons; Tollrian and von Elert, 1994) is much smaller than dextran-tetramethylrhodamin (10,000 MW), the kairomone should be able to enter the body much more freely than the dye during embryonic stage 4 .

The mechanism of kairomone reception during this putative developmental window remains an open question. Barry (2002) showed that the development of Chaoborus-induced neckteeth in $D$. pulex is affected by drugs that act on hormone-secreting neurosecretory cells, and Weiss et al. (2012) showed that cholinergic stimulation mediates signal transmission of Chaoborus cues leading to morphological defenses. Besides, Miyakawa et al. (2010) found that genes in the juvenile hormone and the insulin signaling pathways are upregulated in kairomone-treated first-instar juveniles.
Figure 7 illustrates a hypothetical model for necktooth development. After the embryo receives the kairomone stimulus, epidermal cells lining the future necktooth region proliferate intensely in the postembryonic instars. These proliferated cells then secrete the cuticle of spikes, and epidermal cells underlining the spikes enlarge as a loose connective tissue, leading to thickening of the crest. In the crest, some epidermal cells may lose cell-cell adhesion and dramatically increase their cytoplasmic volume, leading to the swollen occipital region.

Among Daphnia species, crest or helmet growth in the defensive morph is positioned in various regions of the head. These differences across taxa could be established by the same induction and cell proliferation mechanisms as in $D$. pulex, with variation in the form and position of defensive structures determined by downstream regulatory genes specifying the position and pattern of cell proliferation.

The effect of $\mathrm{LiCl}$ on crest formation suggests that this

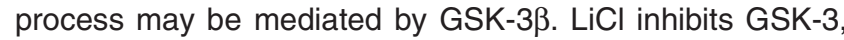
allowing $\beta$-catenin to accumulate and affect the transcription of Wnt target genes (Klein and Melton, 1996). Our results suggest the involvement of Wnt signaling in crest development. Twelve Wnt subfamilies have been identified in $D$. pulex (Janssen et al., 2010), but using real-time PCR we have so far failed to observe Wnt gene expression during production of the defensive phenotype. Future studies using in situ hybridization and RNA interference (Kato et al., 2011) will be needed to show the direct involvement of the Wnt 
pathway in defense morph formation.

\section{ACKNOWLEDGMENTS}

We thank Dr. Toru Miura and his lab members for comments and suggestions; Drs. Shin-ichiro S. Matsuzaki, Maki Imai, and Hitoshi Miyakawa for their assistance in field sampling; Dr. Junichi Obokata for providing C. reinhardtii; Dr. Matthew H. Dick for critical comments; and members of the Tochinai laboratory for helpful advice and discussions.

\section{REFERENCES}

Barry MJ (2002) Progress toward understanding the neurophysiological basis of predator-induced morphology in Daphnia pulex. Physiol Biochem Zool 75: 179-186

Beaton MJ, Hebert PDN (1997) The cellular basis of divergent head morphologies in Daphnia. Limonol Oceanogra 42: 346-356

Colbourne JK, Pfrender ME, Gilbert D, et al. (2011) The ecoresponsive genome of Daphnia pulex. Science 331: 555-561

Cristescu MEA, Colbourne JK, Radivojac J, Lynch M (2006) A microsatellite-based genetic linkage map of the waterflea, Daphnia pulex: On the prospect of crustacean genomics. Genomics 88: 415-430

Dodson SL, Cáceres CE, Rogers DC (2009) Cladocera and other branchiopoda. In "Ecology and classification of North American freshwater invertebrates, third edition" Ed by JH Thorp and AP Covich, Academic Press, London, pp 774-828

Gilbert SF (2010) Developmental Biology. 9th ed, Sinauer Associates Inc, USA

Goto S, Hayashi S (1999) Proximal to distal cell communication in the Drosophila leg provides a basis for an intercalary mechanism of limb patterning. Development 126: 3407-3413

Grasswitz TR, Jones GR (2002) Chemical Ecology. Encyclopedia of Life Sciences. John Wiley \& Sons Inc, USA

Havel JE, Dodson SI (1987) Reproductive costs of Chaoborusinduced polymorphism in Daphnia pulex. Hydrobiologia 150: 273-281

Imai M, Naraki Y, Tochinai S, Miura T (2009) Elaborate regulations of the predator-induced polyphenism in the water flea Daphnia pulex: kairomone-sensitive periods and life-history tradeoffs. J Exp Zool Part A 311: 788-795

Janssen R, Le Gouar M, Pechmann M, et al. (2010) Conservation, loss, and redeployment of Wnt ligands in protostomes: implications for understanding the evolution of segment formation. BMC Evol Biol 10: 374

Jenner RA, Wills MA (2007) The choice of model organisms in evodevo. Nat Rev Genet 8: 311-319

Kato Y, Shiga Y, Kobayashi K, et al. (2011) Development of an RNA interference method in the cladoceran crustacean Daphnia magna. Dev Genes Evol 220: 337-345
Klein PS, Melton DA (1996) A molecular mechanism for the effect of lithium on development. Proc Natl Acad Sci USA 93: $8455-8459$

Krueger DA, Dodson SI (1981) Embryological induction and predation ecology in Daphnia pulex. Limonol Oceanogr 26: 219-223

Laforsch C, Tollrian R (2000) A new preparation technique of daphnids for scaning electron microsopy using hexamethyldisilazane. Arch Hydrobiol 149: 587-596

Laforsch C, Tollrian R (2004) Embryological aspects of inducible morphological defenses in Daphnia. J Morphol 262: 701-707

Miura T (2005) Developmental regulation of caste-specific characters in social-insect polyphenism. Evol Dev 7: 122-129

Miyakawa H, Imai M, Sugimoto N, et al. (2010) Gene up-regulation in response to predator kairomones in the water flea, Daphnia pulex. BMC Dev Biol 10: 45

Parejko K (1992) Embryology of Chaoborus-induced spines in Daphnia pulex. Hydrobiologia 231: 77-84

Sagawa K, Yamagata H, Shiga Y (2005) Exploring embryonic germ line development in the water flea, Daphnia magna, by zincfinger-containing VASA as a marker Gene Expr Patterns 5: 669-678

Spanier KI, Leese F, Mayer C, et al. (2010) Predator-induced defences in Daphnia pulex: Selection and evaluation of internal reference genes for gene expression studies with real-time PCR. BMC Mol Biol 11: 50

Stollewerk A (2010) The water flea Daphnia - a "new" model system for ecology and evolution? J Biol 9: 21

Sueoka N (1960) Mitotic replication of deoxyribonucleic acid in Chlamydomonas reinhardi. P Natl Acad Sci USA 46: 83-91

Suzuki Y, Nijhout HF (2006) Evolution of a polyphenism by genetic accommodation. Science 311: 650-652

Tollrian R (1993) Neckteeth formation in Daphnia pulex as an example of continuous phenotypic plasticity: morphological effects of Chaoborus kairomone concentration and their quantification. $J$ Plankton Res 15: 1309-1318

Tollrian R, Leese F (2010) Ecological genomics: steps towards unraveling the genetic basis of inducible defenses in Daphnia. BMC Biol 8: 51

Tollrian R, Harvell CD (1999) The Ecology and Evolution of Inducible Defenses. Princeton University Press, Princeton

Tollrian R, von Elert E (1994) Enrichment and purification of Chaoborus kairomone from water: Further steps toward its chemical characterization. Limonol Oceanogra 39: 788-796

Weiss LC, Kruppert S, Laforsch C, Tollrian R (2012) Chaoborus and Gasterosteus anti-predator responses in Daphnia pulex are mediated by independent cholinergic and gabaergic neuronal signals. PLoS ONE 7: e36879

(Received October 16, 2012 / Accepted March 11, 2013) 